\title{
A Study of Parameters Setting of the STADZT
}

\author{
Václav Turoň \\ Dept. of Circuit Theory, Czech Technical University, Technická 2, 16627 Praha, Czech Republic
}

Corresponding author: turonvac@fel.cvut.cz

\begin{abstract}
This paper deals with the new time-frequency Short-Time Approximated Discrete Zolotarev Transform (STADZT), which is based on symmetrical Zolotarev polynomials. Due to the special properties of these polynomials, STADZT can be used for spectral analysis of stationary and non-stationary signals with the better time and frequency resolution than the widely used Short-Time Fourier Transform (STFT). This paper describes the parameters of STADZT that have the main influence on its properties and behaviour. The selected parameters include the shape and length of the segmentation window, and the segmentation overlap. Because STADZT is very similar to STFT, the paper includes a comparison of the spectral analysis of a non-stationary signal created by STADZT and by STFT with various settings of the parameters.
\end{abstract}

Keywords: spectral analysis, Zolotarev transform, Fourier transform, spectrogram.

\section{Introduction}

Spectral analysis is a complex field of signal processing which usually deals with transforming the signal between the time domain and the frequency domain. One of the main aims of this analysis is to detect and observe signal information which is not easily analysed in the time domain. Many methods are utilized for this purpose, e.g. the Short-Time Fourier Transform (STFT), the Wavelet Transform (WT), the Hilbert-Huang Transform (HHT) and the novel Short-Time Approximated Discrete Zolotarev Transform (STADZT). All of these methods have certain advantages and disadvantages. STFT is widely used because it is effectively evaluated by the Fast Fourier Transform (FFT), and because of the intuitive interpretation of the results in the form of spectrograms, which give the signal energy in the timefrequency domain [1]. WT is based on the correlation of an analysed signal and wavelet function which is scaled and time dilated [3]. The outcome of WT is a scalogram, which represents the signal energy in the time-scale domain. The interpretation of a scalogram is not as clear as the interpretation of a spectrogram. HHT exploits the decomposition of the signal into the sum of the sub-signals called the Intrinsic Mode Function (IMF) by the process of Empirical Mode Decomposition (EMD). Due to EMD, the complex envelope of the signal can be evaluated by the Hilbert transform (HT) of IMF with higher accuracy than the application of HT to the raw signal [4]. The main disadvantage of HHT lies in the non-intuitive representation of its results. The STADZT transform is very similar to STFT, the difference between being that STADZT is based on selective Zolotarev polynomials, which improve the time and frequency resolution [9]. The result of STADZT shows the signal energy in the time-frequency domain, as well as the spectrogram created by STFT.

All methods have the same difficulty: the parameters must be set and kept for the analysis of the whole signal. This issue is not limiting for the analysis of a stationary signal, because the signal parameters do not change in time. Difficulties arise when the input signal is non-stationary, because many methods must adapt their parameters to obtain a result that can be used for further analysis. These requirements have led to the development of adaptive methods, e.g. STFT with different length of the spectrograms based on various principles, e.g. Minimum Energy of Spectral Leakage (MESP) [6], or the Katkovnik algorithm, which utilizes the actual frequency of the analysed signal [7].

This paper focuses on setting the parameters for STADZT. This is a new method in the field of spectral analysis, and no study has yet been performed that systematically describes its parameters. The next part of the paper briefly introduces the fundamental principle of STADZT. The main part describes the parameters and discusses their influence on the time and frequency resolution of STADZT. The well-known STFT serves as a benchmark for greater clarity of the examples that are presented.

\section{Approximated discrete Zolotarev transform}

The Approximated Discrete Zolotarev Transform (ADZT) is a new time-frequency method that was specially designed by Radim Špetík [9] for spectral analysis of non-stationary signals. The transform is based on symmetrical Zolotarev polynomials that create its basis function. These polynomials can be expressed 
as a weighted sum of Chebyshev polynomials of the first and second kind, and it can be written using cosine and sine notation, respectively [13]. Thus, the basis function can be defined as [9]

$$
\begin{aligned}
& \operatorname{zexp}(\ell, i 2 \pi t)=\mathrm{z} \cos (\ell, 2 \pi t)+i \operatorname{zsin}(\ell, 2 \pi t) \\
& =\sum_{\mu=-\ell}^{\ell} a_{2 \mu}^{\prime}(\kappa) \cos (2 \pi \mu t) \\
& \quad+i \sum_{\mu=-\ell}^{\ell} b_{2 \mu-1}^{\prime}(\kappa) \sin (2 \pi \mu t) \\
& =\sum_{\mu=-\ell}^{\ell} c_{2 \mu}^{\prime}(\kappa) \exp (2 \pi \mu t),
\end{aligned}
$$

where $\ell$ denotes the required degree of the polynomials and $\kappa$ signifies the selectivity, which is closely related to the height of the central lobes. A stable recursive algorithm for computing the coefficients $a_{2 \mu}^{\prime}, b_{2 \mu}^{\prime}$ and $c_{2 \mu}^{\prime}$ is given in [13].

From the spectral point of view, the basis functions can be separated to the stationary $S(k)$ and nonstationary $N(k)$ parts

$$
S_{Z}(k)=v_{k} \mathcal{S}(k)-\left(1-v_{k}\right) \mathcal{N}(k),
$$

where $v_{k}$ is a weighted factor [5]. Because the evaluation of the coefficients of Zolotarev polynomials $a_{2 m}^{\prime}, b_{2 m}^{\prime}$ is rather tedious, work [9] suggested an algorithm based on the minimization of the non-stationary part of Zolotarev polynomials. It can be construed as a filtering or rearrangement of the Fourier spectra according to

$$
\mathbf{S}_{Z}=\mathbf{Z} \cdot \mathbf{S}
$$

where $\mathbf{S}_{Z}$ and $\mathbf{S}$ correspond to the coefficients of the Zolotarev and Fourier spectra, respectively. The matrix $\mathbf{Z}$ contains coefficients of selective Zolotarev polynomials $c_{2 \mu}^{\prime}$ which are optimally chosen in compliance with an analysed signal [9].

The time-frequency analysis of the signal is realized by the STADZT, which is evaluated in the similar way as the STFT. However, instead of the exponential function the Zolotarev basis zexp 1 is used. Thus, the zologram can be defined as [10]

$$
S_{Z}(\ell, n)=\sum_{m=-\infty}^{\infty} s(m) w(m-n) \operatorname{zexp}(\ell, i 2 \pi n)
$$

where $w(m)$ must be the final length window resulting in signal segmentation. Thus all parameters of STADZT are the same as the parameters of STFT. The following part of paper deals with the impact of parameter settings on the time and frequency resolution of STADZT.

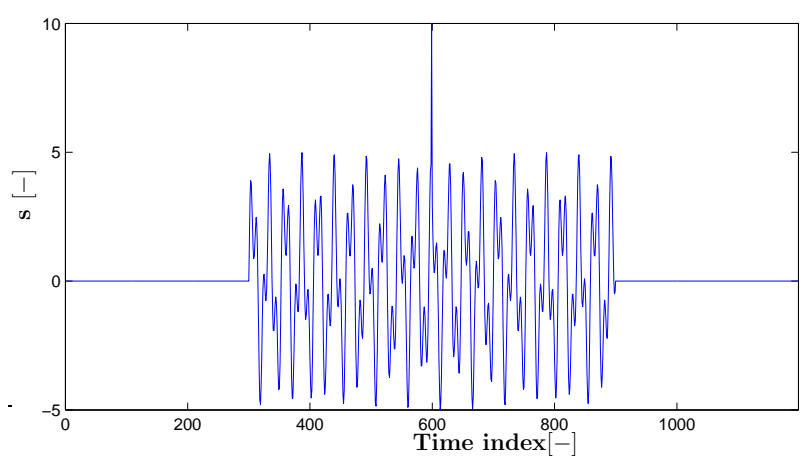

Figure 1: Analysed signal is composed of the sum of two harmonic waves and an unit impulse.

\section{Transform parameters}

The right choice of parameters has the main impact on the time and frequency resolution of the transforms that is used - STFT or STADZT. There are three parameters which closely relate to this issue - window shape, window length and segment overlap. The following part of paper provides a short description of these selected parameters and shows their influence on the spectral analysis of a non-stationary signal. The analysed signal is composed of the sum of two harmonic waves $s_{1}=\cos \left(2 \pi n \frac{k_{1}}{N}\right)$ and $s_{2}=\cos \left(2 \pi n \frac{k_{2}}{N}\right)$, where parameter $n \in(1, N), N=1200, k_{1}=75$ and $k_{2}=190$, and unit impulse which is situated at the center of signal (see Fig. 1).

\subsection{Window shape}

The main difficulty with the widely used Discrete Fourier Transform (DFT) is the presence of spectral leakage, which limits the frequency resolution of STFT. The spectral leakage arises when the orthogonality between the analysed signal and the basis function (the complex exponential in this case) is violated. From another point of view, the leakage originates from the shortening signal to the finite length by the weighting function or segmentation window, respectively. As a consequence of shortening, the spectrum of the analysed signal is given as the result of the convolution of the spectral coefficients of the signal and the weighting function [11], [2]. The measure of leakage depends on the following factors:

- the shape of the segmentation window;

- the rate of segment length and the period of the signal.

The first parameter of interest is the shape of the segmentation window, because it has a direct influence on the frequency resolution of STFT. A segmentation window with a smoothly rising and falling edge suppresses the spectral leakage more effectively than a window with sharp edges. When a smooth window is 


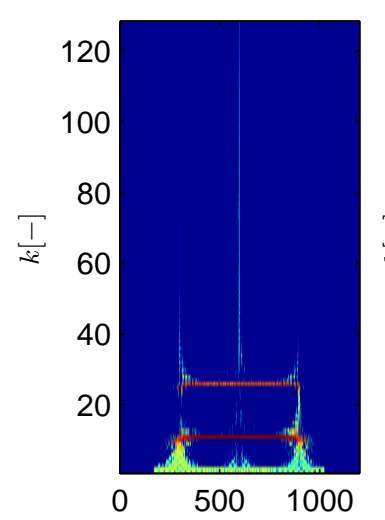

a) Time index $[-]$

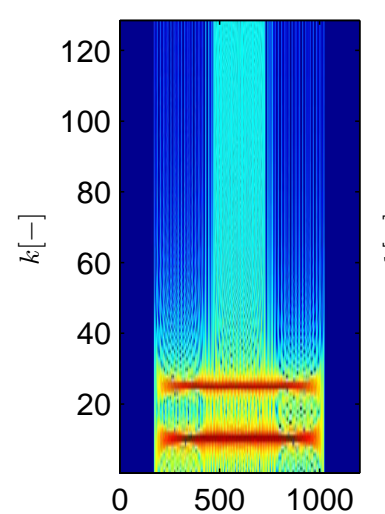

e) Time index $[-]$

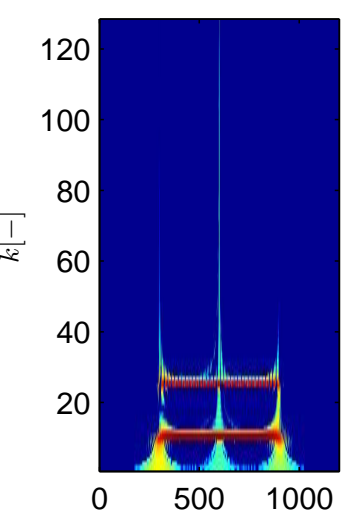

b) Time index $[-]$

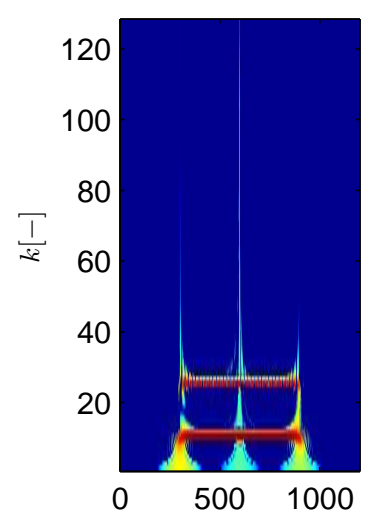

c) Time index $[-]$

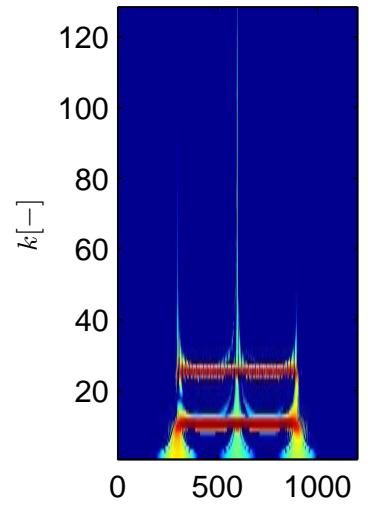

d) Time index $[-]$

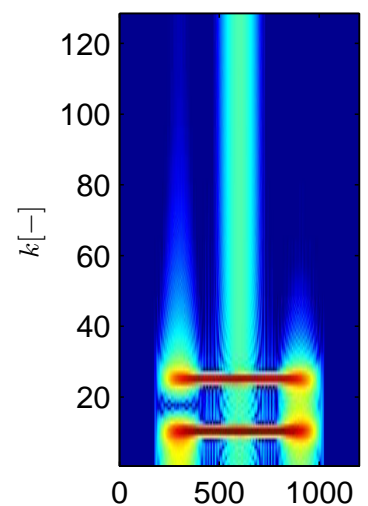

f) Time index $[-]$

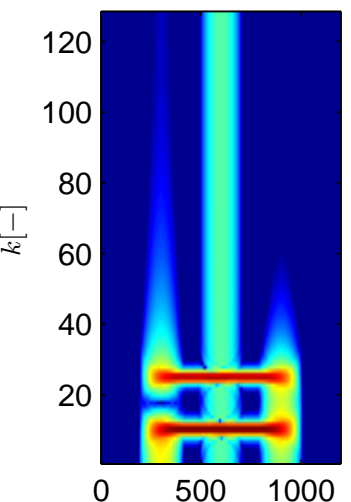

g) Time index $[-]$

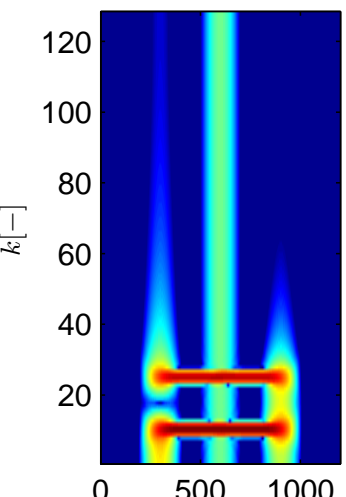

h) Time index $[-]$

Figure 2: STADZT zolograms created by the various window shape: a) Rectangular, b) Hamming, c) Hann, and d) Blackman. STFT spectrograms created by the various window shape: e) Rectangular, f) Hamming, g) Hann, and h) Blackman.

applied to the analysed signal, the discontinuities at the boundary of the periodic extension are reduced. Thus the frequency information of the main signal components is more distinguishable from each other signal component. The most frequent windows are Hamming, Hann and Blackman windows. More information about the application of segmentation windows can be found in [11], [2].

Fig. 2 contains a comparison of the STFT transform and the STADZT transform with various shapes of the segmentation window. The other parameters are the same for both transforms: the window length is 256 samples and the segment overlap is 255 samples. As is shown in Figs. 2e-h, the spectral leakage of STFT is suppressed by using a smooth window - the best suppression is achieved by the Blackman window because the side lobe level is $58 \mathrm{~dB}$ down [2]. However, the best frequency resolution for STADZT is obtained for a rectangular window (see Figire 2a). When some other window shape is applied, the interference between the window and the ADZT time selective basis deteriorates the performance of ADZT algorithm, which evaluates the optimal coefficients of the Zolotarev basis according to the actual segment of the signal (see Figures $2 \mathrm{a}-\mathrm{d}$ ).

\subsection{Window length}

The next parameter that has a notable effect on frequency and time resolution is the length of the segmentation window. The spectral resolution of DFT is defined as

$$
\Delta f=\beta \frac{f_{s}}{N},
$$

where $f_{s}$ is the sampling frequency, $N$ is the length of the signal, and $\beta$ reflects the equivalent noise bandwidth of the window shape that is used [2]. It is apparent that the frequency resolution increases with the prolonged segment. However, it is limited by the HeisenbergGabor uncertainty principle, which states that it is not possible to get the best frequency resolution without losing the time resolution, and vice versa [1]. For this reason, the compromise between time and frequency resolution must be set for STFT. This property is demonstrated by the spectrograms created by STFT with increasing window length (see Figures $3 \mathrm{e}-\mathrm{h}$ ). The best time resolution is obtained for the shortest window length - all sudden changes of signal are located with high accuracy in time, but the frequency resolution is rather poor (see Figure 3e). When the longest window is applied, the frequency resolution is increased but 


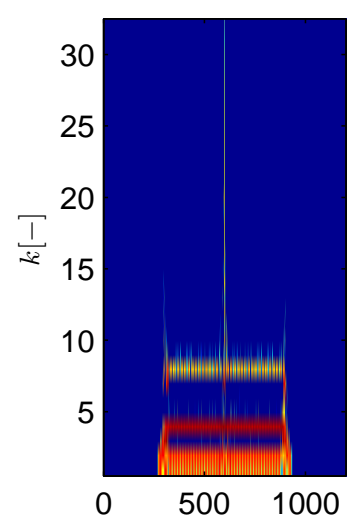

a) Time index $[-]$

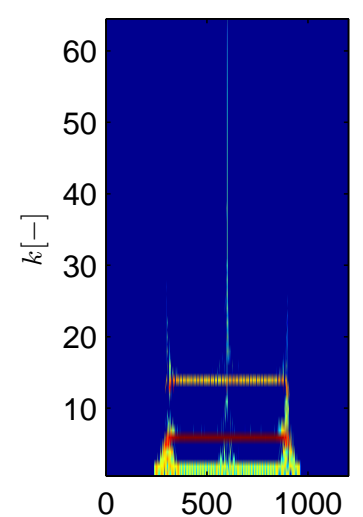

b) Time index $[-]$

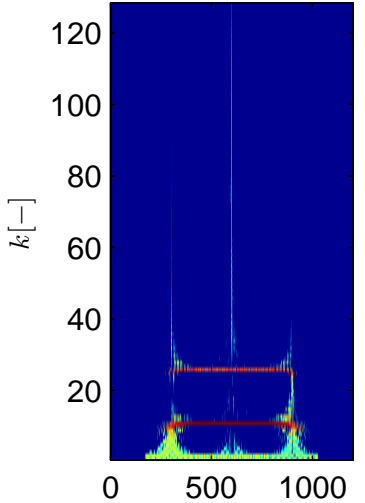

c) Time index $[-]$

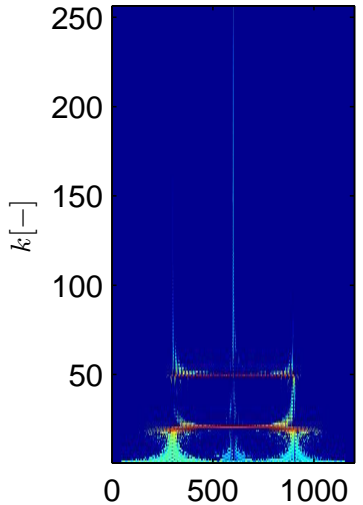

d) Time index $[-]$
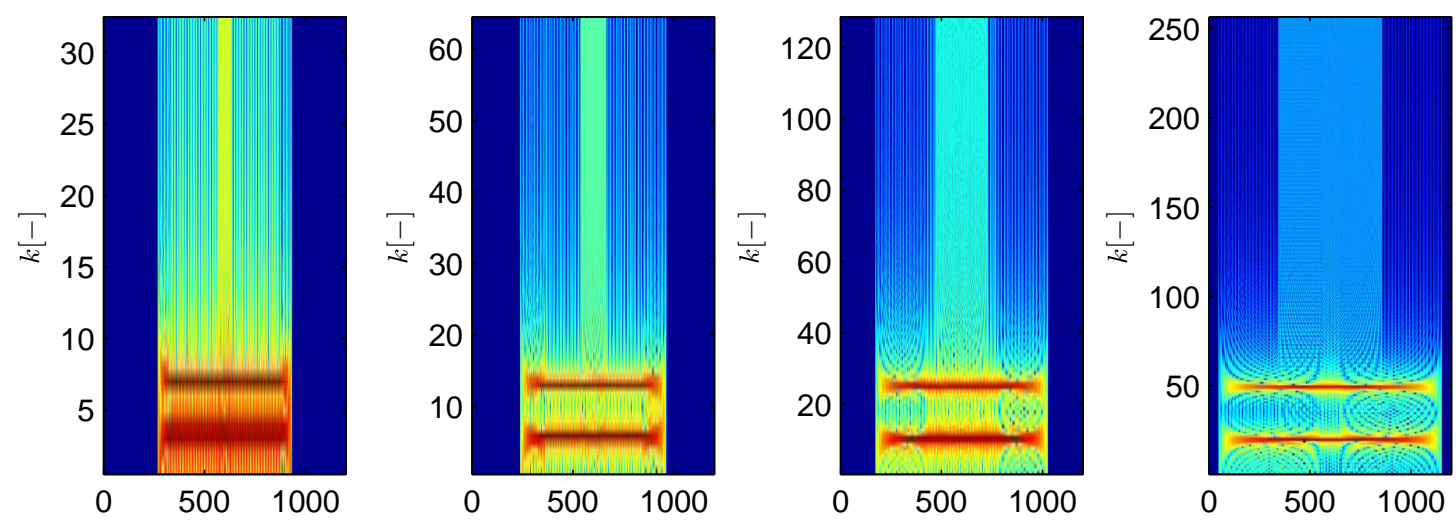

g) Time index $[-]$

h) Time index $[-]$

Figure 3: STADZT zolograms created by the various window length a) 64 samples, b) 128 samples, c) 256 samples, and d) 512 samples. STFT spectrograms created by the various window shape: e) 64 samples, f) 128 samples, g) 256 samples, and h) 512 samples.

the time localization of the signal deteriorates (see Figure 3f).

The frequency resolution of STADZT is given by the window length as well as STFT, but the time resolution is not constant for all frequencies. The reason for this behaviour is that high frequencies can be better analysed than low frequencies by high-order Zolotarev polynomials [10]. One advantage of STADZT is that the time resolution and the frequency resolution are independent from each other in a certain manner, because optimal coefficients of the symmetrical Zolotarev polynomials are set by the ADZT algorithm. Figures 3ad illustrate the good time resolution of STADZT, which is not significantly corrupted by increasing window length. Only the frequency resolution is ameliorated.

It is worth noting that all spectrograms in Figures 3 and 4 are created by STFT using a rectangular window. The first reason for this choice is that ADZT achieves the best results for a rectangular window (see Figure 3.1). The second reason is that the principle of ADZT can be understood as filtering of Fourier spectra. So when we use a rectangular window, we get an original spectrogram which is processed by STADZT.

\subsection{Segment overlap}

The last described parameter is the overlap of the signal segments. If non-overlap segments are used, STFT will lose information about the signal near the boundaries because of the window shape that is applied [2]. STFT therefore usually uses an overlap of $50 \%$ or $75 \%$, which enables the whole signal to be analysed without significant loss of information. Another advantage is that it reduces the extra effort for computing the Fourier spectra for segments with an overlap of $99 \%$.

The evaluation of the optimal Zolotarev coefficients $\mathbf{Z}$ is based on minimizing the non-stationary part of Zolotarev polynomials represented by the spectral coefficients of complex Fourier spectra (2) [5]. As a consequence, the ADZT algorithm is very sensitive to the phase of the input signal. The best time resolution is obtained for overlap $99 \%$, which approximately relates to a segmentation step of 1 sample in this case (see Figure 4). This property is shown in Figures $4 a-d$ ), which illustrate the zolograms created by STADZT with decreasing overlap. The worst case for time resolution is achieved for an overlap of $50 \%$, when the depicted zologram does not contain information about the unit 


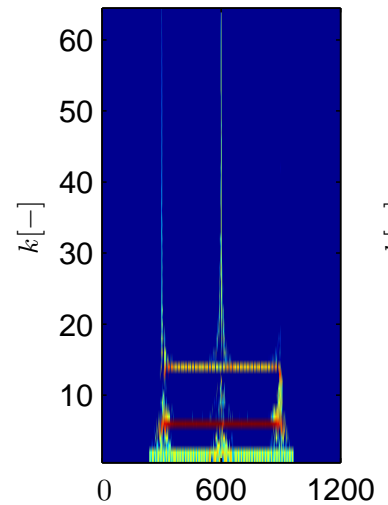

a) Time index $[-]$

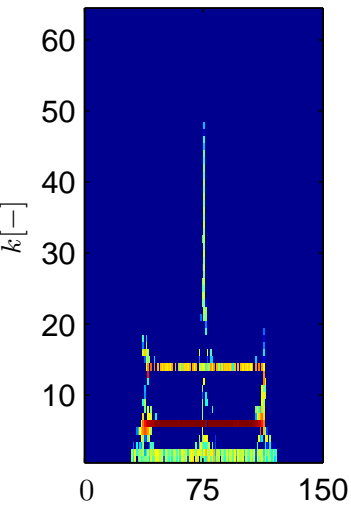

b) Time index $[-]$

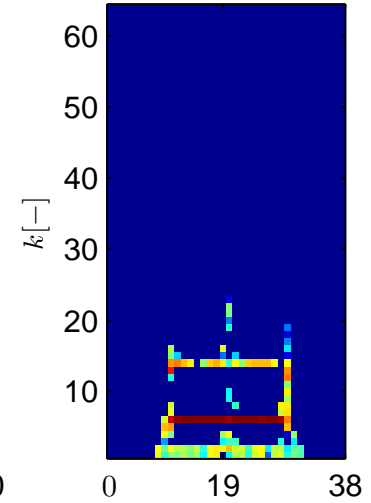

c) Time index $[-]$

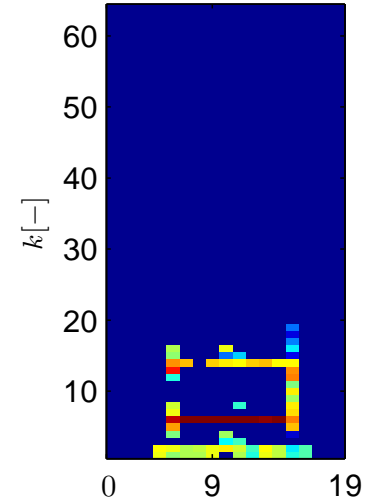

d) Time index $[-]$

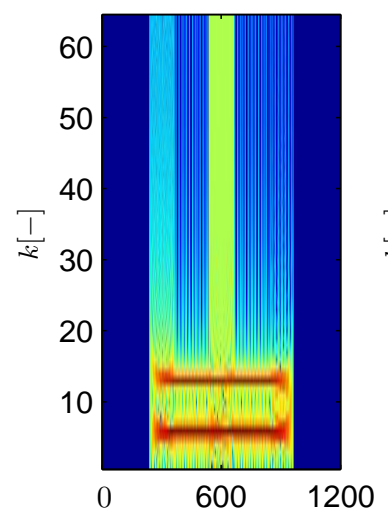

e) Time index $[-]$

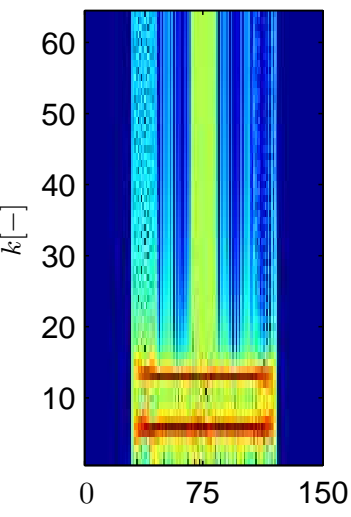

f) Time index $[-]$

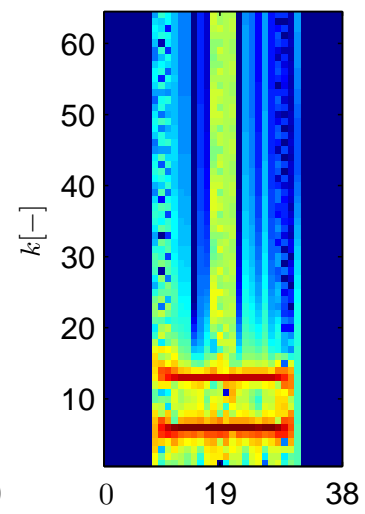

g) Time index $[-]$

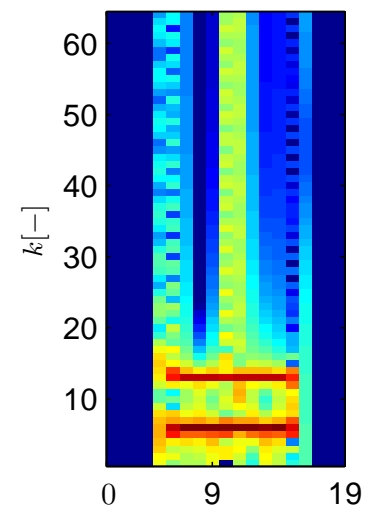

h) Time index $[-]$

Figure 4: STADZT zolograms created by window length 128 samples and various overlap a) 127 samples $~ 99 \%$ overlap, b) 120 samples $\sim 93 \%$ overlap, c) 96 samples $\sim 75 \%$ overlap, and d) 64 samples $\sim 50 \%$ overlap. STFT spectrograms created by window length 128 samples and various window shape: e) 127 samples $\sim 99$ percent overlap, f) 120 samples $\sim 99 \%$ overlap, g) 96 samples $\sim 75 \%$ overlap, and h) 64 samples $\sim 99 \%$ overlap.

impulse (see Figure 4d).

\section{Conclusion}

This paper has presented a study of the parameters which affect the time-frequency analysis created by the new STADZT transform. The parameters discussed here include the shape and the length of the segmentation window, and the segment overlap. These parameters are crucial for STFT, where the compromise between time resolution and the frequency resolution must be set. However, STADZT is not so sensitive to these parameters, because the coefficients of the basis function created by the selective Zolotarev polynomials are adjusted for the analysed signal. Due to these basis functions, the best frequency resolution is acquired for a rectangular window, which is not good for analysis by STFT. In the case of spectral leakage reduction by using the window function, the resulting STFT spectrogram contains less information that can be used for adjusting the ADZT basis functions. As a consequence, the STADZT zologram (see Figures $2 \mathrm{~b}-\mathrm{d}$ ) is worse than the zologram using a rectangular window (see Figire 2a).
The next benefit of the Zolotarev basis is that the time resolution of STADZT does not depend strictly on the window length. Greater window length means more information in the STFT spectrogram, which can be used for optimal adjustment of the Zolotarev basis function. Hence, STADZT is able to obtain good time and frequency resolution simultaneously (see Figure 3d). A substantial feature of STADZT is that the time resolution is not constant for the whole frequency range. Thus the time instants of signal changes are localized with higher accuracy for high frequencies than for low frequencies.

More information about Zolotarev polynomials and their application can be found on the website [12].

\section{Acknowledgements}

The research presented in this paper was supervised by Prof. P. Sovka, FEE CTU in Prague, and by Prof. M. Vlček, FTS CTU in Prague. The author wishes to thank the Grant Agency of the Czech Republic for supporting this research through project P-102/11/1795: 
Novel selective transforms for non-stationary signal processing.

\section{References}

[1] R. E. Croichiere. A weighted overlap-add method of short-time fourier analysis/synthesis. IEEE Transactions on Acoustics, Speech, and Signal Processing, 28(1):99-102, 1980.

[2] F. J. Harris. On the use of windows for harmonic analysis with the descrete transform. Proceeding of the IEEE 66(1):51-83, 1978.

[3] C. Heil, D. F. Walnut, I. Daubechies. Fundamental papers in wavelet theory. Princeton University Press, Princeton, 2006.

[4] E. N. Huang, Z. Shen, S. R. Long et al. The empirical mode decomposition and the Hilbert spectrum for nonlinear and non-stationary time series analysis, Proc. R. Soc. London 454:903-995, 1998.

[5] J. Janik, V. Turon, P. Sovka et al. A way to a new multi-spectral transform. In 11th WSEAS International Conference on Signal Processing, Computational Geometry and Artificial Vision, Florence, 2011.

[6] A. Lukin, J. Todd. Adaptive time-frequency resolution for analysis and processing of audio. In Audio Engineering Society 120th Convention, Paric, 2006.
[7] V. Katkovnik, L. Stankovic. Periodogram with varying and data-driven window length. Signal Processing 67(3):345-358, 1998.

[8] A. V. Oppenhein, R. W. Schafer, J. R. Buck. Discrete-time signal processing. 2nd ed., PrenticeHall, New York, 1999.

[9] R. Spetik. The discrete Zolotarev transform. FEE CTU in Prague, Prague, 2009.

[10] V. Turon, J. Janik, P. Sovka et al. Study of ADZT properties for spectral analysis. In 11th WSEAS International Conference on Signal Processing, Computational Geometry and Artificial Vision, Florence, 2011.

[11] J. Uhlir, P. Sovka, R. Cmejla. Uvod do cislicoveho zpracovani signalu. FEE CTU in Prague, Prague, 2003.

[12] M. Vlcek et al. Novel selective transforms for non-stationary processing. http://amber.feld. cvut.cz/selective\%20transforms [2012-09-25].

[13] M. Vlcek, R. Unbehaen. Zolotarev polynomials and optimal FIR filters. IEEE Transaction on Signal Processing 47(3):717-730, 2006. 